\title{
Cytoplasmic Organelle
}

National Cancer Institute

\section{Source}

National Cancer Institute. Cytoplasmic Organelle. NCI Thesaurus. Code C13269.

Cell part which consists of macromolecules aggregated into discrete structures in the protoplasm. (Digital Anatomist Foundational Model) 